\section{Age at onset and cognitive functioning}

\section{in schizophrenia}

\author{
ANNAMARI TUULIO-HENRIKSSON, TIMO PARTONEN, JAANA SUVISAARI, \\ JARI HAUKKA and JOUKO LÖNNQVIST
}

\begin{abstract}
Background Impairments in cognitive functioning are common in schizophrenia, and the degree of impairment may be associated with the individual's age at onset of the disorder.
\end{abstract}

\begin{abstract}
Aims To examine the effect of age at onset on cognitive functioning using the California Verbal Learning Test, sub-tests from the Wechsler Memory Scale -

Revised and sub-scales from the Wechsler Adult Intelligence Scale - Revised among families with schizophrenia.
\end{abstract}

\begin{abstract}
Method The effect of age at onset on cognitive function in 237 people with schizophrenia from a population-based sample was examined using linear mixed effects models with family as the random effect, and age, gender, chronicity of the illness and number of affected first-degree relatives as fixed effects.
\end{abstract}

Results Impairment in verbal learning and memory was associated with earlier disease onset. No association was found for working memory or IQ.

Conclusions In patients with earlyonset schizophrenia, verbal memory functions in particular should be taken into account in neuropsychological evaluation and efforts at remediation.

\section{Declaration of interest Partial \\ financial support received from \\ Millennium Pharmaceuticals Inc. and \\ American Home Products Corp., \\ Wyeth-Ayerst Research Division.}

Schizophrenia often has its onset in young adulthood, with severe negative consequences on education, social relationships and employment. Early onset has been related to poor clinical and psychosocial outcomes (DeLisi, 1992; Hoff et al, 1996; Eggers \& Bunk, 1997). Moreover, age at onset may be associated with the severity of deficits in cognitive functions, such as learning (Jeste et al, 1998), attention and motor speed, understanding and producing speech (Hoff et al, 1996) and retrieval memory (Paulsen et al, 1995). Using a population-based sample of families in Finland, we set out to study whether the age at onset does associate with performance in attention and working memory, verbal learning and memory, and verbal and visuospatial ability. Following on from previous studies, we examined whether familial occurrence of psychosis, which has been associated with the age at onset (Suvisaari et al, 1998) and with the degree of cognitive deficits (Faraone et al, 2000; Tuulio-Henriksson et al, 2003), contributes to the effect of age at onset on the cognitive functions.

\section{METHOD}

\section{Sample}

The study sample was drawn from families including individuals with schizophreniaspectrum disorders previously recruited for a population-based genetic epidemiological study, described in detail by Hovatta et al (1997), Ekelund et al (2000) and Paunio et al (2001). This study identified 33731 persons $(58 \%$ males) with a diagnosis of schizophrenia, schizoaffective psychosis or schizophreniform disorder from a cohort of all people born in Finland during the period 1940 to 1976 inclusive. Data on the diagnoses were derived from three nationwide computerised health care registers (the Hospital Discharge Register, the Free Medicine Register and the Pension
Register) covering the years 1969-1998. Personal identification numbers, which code date of birth and gender, are unique for each individual; by linking the numbers of the affected subjects to the data on their family members derived from the National Population Register, we were able to construct pedigrees. The protocol was accepted by the ethics committee of the National Public Health Institute, and the study was granted permission by the Ministry of Social Affairs and Health.

Blood samples were drawn from 930 families identified from the registers; they comprised 281 families originating from a genetic isolate in the north-eastern part of Finland, with at least one child with schizophrenia, schizoaffective psychosis or schizophreniform disorder, and 649 families with at least two children with these disorders from the whole country. The collection of blood samples for the molecular genetic studies (Hovatta et al, 1997; Ekelund et al, 2001; Paunio et al, 2001) followed the recommendations given in the Declaration of Helsinki and its amendments. Consent for drawing the blood sample and for a future contact was documented in writing.

All available psychiatric case notes covering the patient's whole illness history were collected for individuals with a diagnosis of schizophrenia, schizoaffective psychosis or schizophreniform disorder in any of the three national registers, and from whom a blood sample was drawn $(n=1410)$. For each case, two psychiatrists, masked to family structure and register diagnosis, assessed independently the bestestimate lifetime diagnoses according to DSM-IV criteria (American Psychiatric Association, 1994). One of the assessors also filled out the Operational Criteria Checklist for Psychotic Illness (OPCRIT; McGuffin et al, 1991). Disagreements on the assessment of research diagnoses were reviewed by a third assessor and a consensus was reached.

All 281 families originating from the genetic isolate, and a sample of 50 families from the whole of Finland randomly selected from families who had previously given blood samples, were asked to participate in this study for detailed diagnostic information and neuropsychological examination. We began by contacting affected individuals (with the permission of their treating physician) to begin the informed consent process. After the affected person had given written consent, the 
rest of the family were contacted and their consent requested, following a complete description of the study.

From the 275 families who gave consent, 411 patients and 561 family members were interviewed using the structured Clinical Interview for DSM-IV (SCID; First et al, 1997). All the interviewers received standardised training in the use of this instrument. The final consensus diagnoses were based on data collected from the records, the OPCRIT assessment and the SCID. The same neuropsychological test battery was administered to both affected and unaffected participants. For each family we endeavoured to interview the same number of affected and unaffected siblings, selecting those closest to the proband's age and of the same gender, if possible.

We excluded 41 patients who had a diagnosis of a schizophrenia-spectrum disorder in the registers but who were assigned a consensus diagnosis of bipolar disorder, and 11 patients who received a consensus diagnosis of a non-psychotic disorder. Furthermore, we excluded 52 patients with schizoaffective disorder and 20 patients with schizophreniform disorder. This left us with 287 patients with schizophrenia. Of these, 35 either did not give a valid test performance or were untestable because of acute psychotic state or severe medical comorbidity. Reliable information about age at illness onset was not received for 11 patients, and 4 were excluded because of the very early age at onset (7-12 years). The cut-off point of age $>12$ years was chosen on the basis of previous research into childhood-onset schizophrenia (for a review, see Nicolson \& Rapoport, 1999).

Our study thus comprised 237 persons with a diagnosis of schizophrenia from 208 families. Of the patients, 81 were women and 156 were men $(P<0.001)$, reflecting a slight overrepresentation of males $(66 \% v .58 \%$ in the registers). The mean ages of women and men were similar, at about 45 years (Table 1 ). The course of the illness was chronic in $71 \%$ of women and $74 \%$ of men (data derived from the OPCRIT).

Information about the age at onset of schizophrenic illness was derived from the case notes as one of the OPCRIT items. The case notes were comprehensive and covered the in-patient and out-patient phases of the whole treatment history. The age at onset was defined as the earliest age at which medical advice was sought
Table I Demographic characteristics of the participants with schizophrenia $(\boldsymbol{n}=\mathbf{2 3 7})$

\begin{tabular}{llll} 
Characteristic & & \\
\hline Gender & 34 \\
$\quad$ Female, \% & & \\
Age at illness onset, years: mean (s.d.) & & \\
$\quad$ Women & 23.0 & $(5.7)$ \\
Men & 23.3 & $(5.7)$ \\
All & 23.2 & $(5.7)$ \\
Age, years: mean (s.d.) & & \\
$\quad$ Women & 45.4 & $(8.4)$ \\
Men & 45.0 & $(7.5)$ \\
All & 45.2 & $(7.8)$ \\
Education, years: mean (s.d.) & 10.0 & $(2.4)$ \\
Estimated IQ: mean (s.d.) & 82.9 & $(15.9)$ \\
\hline
\end{tabular}

for occurrence of psychiatric symptoms, or at which the symptoms began to cause subjective distress or impair functioning. The mean age at onset in the final sample was 23.2 years with no difference between women and men (Table 1), which is in line with a previous epidemiological study in Finland (Suvisaari et al, 1998). The IQ was estimated from four sub-scales from the Wechsler Adult Intelligence Scale Revised (WAIS-R; Wechsler, 1981).

\section{Neuropsychological tests}

The neuropsychological tests were administered to all participants in a fixed order. All examiners were psychologists or advanced psychiatric nurses extensively trained and supervised with the test battery. Experienced psychologists scored all the tests using the scoring rules detailed in the test manuals.

The test procedures have been described in detail by Tuulio-Henriksson et al (2002). Auditory attention was assessed with the Digit Span Forward task, and verbal working memory with the Digit Span Backward task of the Wechsler Memory Scale - Revised (WMS-R; Wechsler, 1987). According to Finnish normative data, the test-retest reliability coefficients of the Digit Span sub-tests vary with age from 0.74 to 0.82 (Wechsler, 1996). The Visual Span Forward sub-test of the WMS-R (Wechsler, 1987) was used to assess visual attention and the Backward condition of the span task was used to measure visuospatial working memory. According to Finnish normative data, the test-retest reliability coefficients of the Visual Span sub-tests vary with age from 0.72 to 0.80 (Wechsler, 1996).

Verbal learning and memory were assessed with the California Verbal Learning Test (CVLT; Delis et al, 1987), which examines recall and recognition of word lists over a number of trials. We report the following variables derived from the test: total recall (learning and memory), semantic clustering, learning from the beginning of the list (primacy) and from the end (recency), recall errors (perseverations and intrusions) and recognition memory (discriminability). No reliability data for Finnish subjects exist, but the split-half reliability of the CVLT is $0.77-$ 0.86 , according to the test manual (Delis et al, 1987).

Four sub-tests of the WAIS-R were used for measuring IQ. Verbal ability and abstraction were measured with the Vocabulary and Similarities sub-tests, respectively; the vocabulary test is considered to be the best single measure of general ability (Lezak, 1995). The Block Design and Digit Symbol sub-tests have a motor component, as the trials are timed; the former is a measure of visuospatial reasoning, and the latter measures psychomotor speed. According to Finnish normative data, the test-retest reliabilities for the Vocabulary, Similarities, Block Design and Digit Symbol measures are 0.89-0.95, $0.69-0.88,0.78-0.83$ and $0.82-0.86$, respectively, depending on age (Wechsler, 1992).

\section{Statistical analyses}

The analyses were made using linear mixed effect models separately for each neuropsychological test variable. Age at onset was treated in the models as a continuous variable, ranging from 13 to 44 years. In each model, family was included as a random effect, as from some families more than one family member was included. Age, gender, course of the illness (chronic $v$. episodic) and age at onset (years) were included as the fixed effects. Furthermore, we included in the models a continuous variable of the number of affected members in the family as a fixed effect in order to detect whether familial loading, measured by the number of first-degree relatives with a psychotic disorder, modifies the effect of the age at onset on cognitive functions. The information concerning the illness of the first-degree relatives was obtained from 
the national registers. In addition, we remodelled all test variables with the abovementioned random and fixed effects plus duration of the illness (tertiles from age at onset subtracted from the age at testing, in years). All analyses were two-tailed, and the probability level of $P<0.05$ indicated statistical significance. Analyses were performed using the S-Plus statistical software, version 3.4 (S-Plus, 1996).

\section{RESULTS}

\section{Effect of age at onset}

In the linear mixed effect models, lower scores on four cognitive functions were significantly associated with earlier age at onset (Table 2). These functions were verbal learning and memory, assessed as total recall from trials 1 to 5 in the CVLT (coefficient for the effect of how much each increasing year in the age at onset changes the value of the test score 0.483 , s.d. $=0.14, \quad P=0.002) ; \quad$ using semantic clusters as a learning strategy (effect coefficient 0.023 , s.d. $=0.01, P=0.02$ ); recognition memory (effect coefficient 0.256 , s.d. $=0.15, P=0.05)$; and making errors

Table 2 Neuropsychological test results and effect of age at onset of schizophrenia

\begin{tabular}{lccc}
\hline Variable & $\begin{array}{c}\text { Test score } \\
\text { Mean (s.d.) }\end{array}$ & $\begin{array}{c}\text { Effect of age at onset, per year } \\
\text { Coefficient (s.d.) }\end{array}$ & $P$ \\
\hline Attention and working memory & & & \\
$\quad$ Auditory attention & $6.0(1.8)$ & $0.022(0.02)$ & 0.31 \\
Verbal working memory & $4.9(1.7)$ & $0.028(0.02)$ & 0.16 \\
Visual attention & $7.4(1.8)$ & $0.019(0.02)$ & 0.38 \\
Visual working memory & $6.5(2.3)$ & $0.023(0.03)$ & 0.38 \\
Verbal memory functions & & & \\
Verbal learning and memory & $33.8(12.6)$ & $0.483(0.14)$ & 0.002 \\
Semantic clustering & $7.2(6.3)$ & $0.023(0.01)$ & 0.02 \\
Discriminability & $86.6(11.6)$ & $0.256(0.13)$ & 0.05 \\
Primacy & $26.3(I 1.4)$ & $-0.095(0.14)$ & 0.49 \\
Recency & $32.0(12.9)$ & $-0.159(0.15)$ & 0.30 \\
Delayed verbal memory & $7.2(3.5)$ & $0.067(0.04)$ & 0.10 \\
Perseverations' & $0.5(0.4)$ & $0.001(0.01)$ & 0.90 \\
Intrusions' & $0.7(0.4)$ & $-0.033(0.01)$ & 0.007 \\
IQ & & & \\
Verbal ability & $31.4(14.2)$ & $0.195(0.14)$ & 0.19 \\
Abstraction & $20.2(6.6)$ & $0.146(0.07)$ & 0.06 \\
Visuospatial reasoning & $20.7(10.6)$ & $0.103(0.12)$ & 0.40 \\
Psychomotor speed & $29.2(12.5)$ & $0.086(0.12)$ & 0.49 \\
\hline
\end{tabular}

Linear mixed effects models with family as a random effect, and fixed-effect adjustments for age, gender, chronicity of the illness and number of affected first-degree relatives.

I. Logarithmised for normality. during recall (intrusions) (effect coefficient -0.033 , s.d. $=0.01, P=0.007)$.

\section{Fixed-effect contributions}

Course of the illness (chronic $v$. episodic) contributed to the measured four IQ functions (all $P$ values $<0.03$ ). Chronicity also showed a significant effect on verbal learning and memory (effect coefficient -6.245 , s.d.=1.8, $P<0.001)$, semantic clustering (effect coefficient -0.287 , s.d. $=0.11$, $P=0.02$ ), delayed verbal memory (effect coefficient -1.723 , s.d. $=0.48, P<0.001$ ), recognition memory (effect coefficient -5.300 , s.d. $=1.62, P=0.002)$ and intrusions (effect coefficient 0.348 , s.d. $=0.14$, $P<0.02$ ), but it did not eliminate the effect of age at onset on these variables. Including duration of the illness in the models did not change the results, and this variable did not contribute significantly to any of the measured cognitive functions. The number of affected first-degree relatives in the family showed a significant effect on visual working memory (effect coefficient -0.27 , s.d. $=0.13, P=0.04$ ), verbal ability (effect coefficient $-1.80, \quad$ s.d. $=0.83, \quad P=0.04$ ) and processing speed (effect coefficient -1.31 , s.d. $=0.61, P=0.03$ ).

\section{DISCUSSION}

An association exists between age at onset of schizophrenia and impaired performance in verbal learning and memory tasks. Those younger at disease onset recalled fewer words in verbal list learning, and while recalling made less use of semantic clusters, that is they did not effectively group the words in the list into categories. Furthermore, they performed worse in recognition, and more frequently made intrusive errors (i.e. produced more words that were not in the original list) than those with a later onset. However, earlier age at onset was not associated with lower scores on working memory tasks or on tests measuring IQ functions.

Familial loading, measured as the number of affected first-degree relatives in the family, did not contribute to the effect of age at onset on cognitive functioning, but showed a significant effect on visual working memory, verbal IQ and processing speed. Chronicity contributed strongly to the cognitive test scores, but did not eliminate the significant effect of age at onset from the verbal learning and memory functions.

\section{Strengths and limitations of the study}

To our knowledge, this study is the first to examine how the age at onset is associated with cognitive function, using a large population-based sample of families of people with schizophrenia. Furthermore, we controlled for the impact of familial loading. This study design allowed us to take into account intrafamilial features in the neuropsychological test data. Our analyses showed that scores in visual working memory and verbal IQ were worse when there were more affected first-degree relatives in the family, irrespective of the age at onset. This result provides further support for the evidence of genetic influence on visual working memory deficits in families with individuals with schizophrenia that has been reported in previous studies (Park et al, 1995; Cannon et al, 2000; Tuulio-Henriksson et al, 2002).

Since the participants with schizophrenia in our study sample had a wide range of age, some of the results might reflect normal deterioration of cognitive 
function with increasing age. However, we controlled for the effect of age in the analysis. Moreover, these participants were not people experiencing a first episode, but individuals with an often chronic course of illness who might have been exposed to noxious effects of the illness or medication, with negative effects on cognitive functions. However, by controlling for chronicity, and in additional models also for the duration of the illness in the analysis, we took at least partial account on these potential confounds. Besides, duration of the illness may not be associated with the severity of cognitive deficits in schizophrenia (Heaton et al, 2001).

Our definition of familiality was based on the number of affected first-degree relatives with a psychotic disorder, instead of using the more sophisticated calculations presented by Verdoux et al (1996) or Lawrie et al (2001). However, since the majority of the families under study had at least two affected family members, and therefore represented familial schizophrenia, we considered that it was adequate to use the number of affected family members as a measure of familial loading in the analyses. Furthermore, the method introduced by Lawrie et al (2001) would have assumed spousal correlation to be 0 , but this was not always the case in the genetic isolate included in our study sample.

\section{Verbal memory as a vulnerable cognitive function in early-onset schizophrenia}

More severe learning and memory impairments, as measured by the total recall score on the CVLT, have been associated with earlier onset of schizophrenia (Jeste et al, 1998). In accordance with the study by Jeste et al (1998), cognitive functions related to verbal learning and memory, particularly to initial encoding, showed the strongest associations of interest in our study. This does not imply a relative impairment of verbal learning and memory compared with the other measured cognitive functions. However, the association between age at disease onset and verbal learning and memory remained even in the presence of chronicity, which accounted for most of the variance of the other cognitive functions. Turetsky et al (2002) identified three memory-delineated subtypes of schizophrenia: based on verbal memory impairment (measured using the CVLT) these subtypes were defined as those conforming to the phenotypes of cortical and subcortical dementia, and those with no impairment. Patients in the group in which the memory deficits (poor total recall scores on the CVLT and frequent intrusive errors) were similar to those observed in cortical dementia had an earlier onset of disease than the unimpaired group (Turetsky et al, 2002). Our results support these findings.

Furthermore, patients with prodromal signs of schizophrenia have been found to show compromised verbal memory (free recall of a word list) measured with the Auditory Verbal Learning Test, which is similar to the CVLT used in our study (Hambrecht et al, 2002). Verbal learning and memory functions may be particularly vulnerable to the developmental process leading to schizophrenia. The poor psychosocial outcome associated with early onset of schizophrenia (Schultz et al, 2000) may be influenced by impairment in the verbal learning and memory functions in particular. The deficits associated with verbal memory functions, inevitably undermining social adaptation and educational possibilities, may predispose individuals to an earlier onset of schizophrenia.

\section{IQ and age at onset}

In line with the prospective study by Fuller et al (2002), we found no association between age at onset and measures of IQ. It may be that low IQ, although a risk factor for schizophrenia (David et al, 1997), is not linked with age at onset. However, our cross-sectional study comprised mostly patients with chronic schizophrenia, and chronicity contributed strongly to the test scores in the four WAIS-R scales. The IQ measures might not have been sensitive enough to show association with age at onset in the presence of a strong contribution of chronicity. In a large study of military conscripts, an association was found between poor intellectual functioning at 18 years of age and onset of schizophrenia about 5 years later (Gunnell et al, 2002). However, when IQ was controlled for in a study of adolescent patients with recent onset of schizophrenia, verbal and general memory impairments were particularly observed (Kravariti et al, 2003).

\section{Implications of the study}

Our results are in agreement with previous studies showing that an early age at onset of schizophrenia is associated with specific rather than global cognitive deficits. In our population-based sample of patients with schizophrenia, this association was found in the verbal learning and memory functions. Verbal memory deficits - known to be highly associated with functional outcome in schizophrenia (Green, 1996) should particularly be taken into account in the neuropsychological evaluation and efforts at remediation in patients with early-onset disorder. Prospective research on verbal memory functions during prodromal phases, and on the predictive value of impairments for disease outcome, is urgently required.

\section{ACKNOWLEDGEMENTS}

We thank all the people who participated in this study. The field workers who collected the data and the research clinicians who read through the case notes are gratefully acknowledged.

\section{REFERENCES}

American Psychiatric Association (1994) Diagnostic and Statistical Manual of Mental Disorders (4th edn) (DSM-IV).Washington, DC: APA.

\section{Cannon, T. D., Huttunen, M. O., Lönnqvist, J., et al (2000) The inheritance of neuropsychological dysfunction in twins discordant for schizophrenia. American Journal of Human Genetics, 67, 369-382.}

David, A. S., Malmberg, A., Brandt, L., et al (1997) IQ and risk for schizophrenia: a population-based cohort study. Psychological Medicine, 27, I3II-1323.

Delis, D. C., Kramer, J. H., Kaplan, E., et al (1987) California Verbal Learning Test Manual (Research Edition). San Antonio, TX: Psychological Corporation/Harcourt Brace.

DeLisi, L. E. (1992) The significance of age at onset for schizophrenia. Schizophrenia Bulletin, 18, 209-215.

Eggers, C. \& Bunk, D. (1997) The long-term course of childhood onset schizophrenia: a 42-year followup. Schizophrenia Bulletin, 23, 105-117.

Ekelund, J., Lichtermann, D., Hovatta, I., et al (2000) Genome-wide scan for schizophrenia in the Finnish population: evidence for a locus on chromosome $7 q 22$. Human Molecular Genetics, 12, 1049-1057.

Ekelund, J., Hovatta, I., Parker, A., et al (200I) Chromosome I loci in Finnish schizophrenia families. Human Molecular Genetics, 10, 1611-1617.

Faraone, S. V., Seidman, L. J., Kremen, W. S., et al (2000) Neuropsychologic functioning among the nonpsychotic relatives of schizophrenia patients: the effect of genetic loading. Biological Psychiatry, 48, 120-126.

First, M. B., Spitzer, R. L., Gibbon, M., et al (1997) Structured Clinical Interview for DSM-IV Axis I Disorders - Clinician Version (SCID-CV). Washington, DC: APA.

Fuller, R., Nopoulos, P., Arndt, S., et al (2002) Longitudinal assessment of premorbid cognitive functioning in patients with schizophrenia through examination of standard scholastic test performance. American Journal of Psychiatry, 159, II83-1189. 
Green, M. F. (1996) What are the functional consequences of neurocognitive deficits in schizophrenia? American Journal of Psychiatry, 153, $321-330$

Gunnell, D., Harrison, G., Rasmussen, F., et al (2002) Associations between premorbid intellectual performance, early life-exposures and early-onset schizophrenia. Cohort study. British Journal of Psychiatry, 181, 298-305.

Hambrecht, M., Lammertink, M., Klosterkötter, J. et al (2002) Subjective and objective neuropsychological abnormalities in a psychosis prodrome clinic. British Journal of Psychiatry, I8I (suppl. 43), s30-s 37.

Heaton, R. K., Gladsjo, J. A., Palmer, B. W., et al (200I) Stability and course of neuropsychological deficits in schizophrenia. Archives of General Psychiatry 58, 24-32.

Hoff, A. L., Harris, D., Faustman, W. O., et al (1996) A neuropsychological study of early onset schizophrenia. Schizophrenia Research, 20, 21-28.

Hovatta, I., Terwilliger, J. D., Lichtermann, D., et a (1997) Schizophrenia in the genetic isolate of Finland. American Journal of Medical Genetics (Neuropsychiatric Genetics), 74, 353-360.

Jeste, D. V., McAdams, L. A., Palmer, B. W., et al (1998) Relationship of neuropsychological and MRI measures to age of onset of schizophrenia. Acta Psychiatrica Scandinavica, 98, 156-164.

Kravariti, E., Morris, R. G., Rabe-Hesketh, S., et al (2003) The Maudsley early onset schizophrenia study: cognitive function in adolescents with recent onset schizophrenia. Schizophrenia Research, 6I, 137-148.

Lawrie, S. M., Whalley, H. C., Abukmeil, S. S., et al (200I) Brain structure, genetic liability, and psychotic symptoms in subjects at high risk of developing schizophrenia. Biological Psychiatry, 15, 8II-823.

Lezak, M. D. (1995) Neuropsychological Assessment (3rd edn). New York: Oxford University Press.

McGuffin, P., Farmer, A. E. \& Harvey, I. (1991) A polydiagnostic application of operational criteria in psychotic illness: development and reliability of the OPCRIT system. Archives of General Psychiatry, 48, 764-770.

Nicolson, R. \& Rapoport, J. L. (1999) Childhoodonset schizophrenia: rare but worth studying. Biological Psychiatry, 46, 1418-1428.

Park, S., Holzman, P. S. \& Goldman-Rakic, P. S. (1995) Spatial working memory deficits in the relatives of schizophrenia patients. Archives of General Psychiatry, 52, $821-828$.

Paulsen, J. S., Heaton, R. K., Sadek, J. R., et al (1995) The nature of learning and memory impairments in schizophrenia. Journal of the International

Neuropsychological Society, I, 88-99.

Paunio, T., Ekelund, J., Varilo, T., et al (200I) Genomewide scan in a nationwide study sample of schizophrenia families in Finland reveals susceptibility loci on chromosomes 2q and 5q. Human Molecular Genetics, I5, 3037-3048.

\section{CLINICAL IMPLICATIONS}

- The association of cognitive deficits (particularly in verbal memory functions) with clinical symptoms and outcome in schizophrenia may vary according to the age at onset of the disease.

- Earlier onset of schizophrenia is associated specifically with verbal learning and memory impairments, independently of familial loading.

- Neuropsychological assessment is important for planning rehabilitation efforts aimed at preventing a poor psychosocial outcome.

\section{LIMITATIONS}

- The participants were not first-episode patients, and it is not known whether they had verbal memory impairment at the time of disease onset.

- Illness factors between disease onset and the time of testing might have contributed to the results.

- As the mean age of the patients was about 45 years, our results may partly reflect the effects of ageing, although we controlled for the effect of age.

ANNAMARI TUULIO-HENRIKSSON, LicPhil, TIMO PARTONEN, MD, PhD, JAANA SUVISAARI, MD, PhD, JARI HAUKKA, PhD, JOUKO LÖNNQVIST, MD, PhD, National Public Health Institute, Department of Mental Health and Alcohol Research, Helsinki, Finland

Correspondence: Annamari Tuulio-Henriksson, National Public Health Institute, Department of Mental Health and Alcohol Research, Mannerheimintie 166, 00300 Helsinki, Finland. Tel: +358947448548; fax: +35894744 8478; e-mail: annamari.tuulio-henriksson@ktl.fi

(First received 28 October 2003, final revision 23 March 2004, accepted 5 April 2004)

\section{Schultz, S. K., Ho, B. C. \& Andreasen, N. C. (2000)} Clinical features characterizing young-onset and intermediate-onset schizophrenia. Journal of Neuropsychiatry and Clinical Neurosciences, 12, 502-505

S-Plus (1996) Version 3.4 for Unix - Supplement. Seattle, WA: MathSoft, Data Analysis Products Division.

Suvisaari, J. M., Haukka, J., Tanskanen, A., et al (1998) Age at onset and outcome in schizophrenia are related to the degree of familial loading. British Journal of Psychiatry, 173, 494-500.

Turetsky, B. I., Moberg, P. J., Mozley, L. H., et al (2002) Memory-delineated subtypes of schizophrenia: relationship to clinical, neuroanatomical, and neurophysiological measures. Neuropsychology, 16 $48 I-490$.

Tuulio-Henriksson, A., Haukka, J., Partonen, T., et al (2002) Heritability and number of quantitative trait loci of neurocognitive functions in families with schizophrenia. American Journal of Medical Genetics (Neuropsychiatric Genetics), II4, 483-490.
Tuulio-Henriksson, A., Arajärvi, R., Partonen, T., et al (2003) Familial loading associates with impairment in visual span among healthy siblings of schizophrenia patients. Biological Psychiatry, 54, 623-628.

Verdoux, H., van Os, J., Sham, P., et al (1996) Does familiality predispose to both emergence and persistence of psychosis? A follow-up study. British Journal of Psychiatry, 168, 620-626.

Wechsler, D. (198I) Wechsler Adult Intelligence ScaleRevised (WAIS-R) Manual. Cleveland, $\mathrm{OH}$ : Psychological Corporation/Harcourt Brace Jovanovich.

Wechsler, D. (1987) Wechsler Memory Scale - Revised (WMS-R) Manual. San Antonio, TX: Psychological Corporation/Harcourt Brace Jovanovich.

Wechsler, D. (1992) WAIS-R Käsikirja [in Finnish] Helsinki: Psykologien Kustannus.

Wechsler, D. (1996) WMS-R Käsikirja [in Finnish] Helsinki: Psykologien Kustannus. 